\title{
A Systematic Scoping Review of the Physical and Mental Wellbeing Needs of Ageing Carers of Individuals with Learning Disabilities within the United Kingdom
}

\author{
Mahon $\mathrm{A}^{1}$, Vseteckova ${ }^{2 *}$, Tilley $\mathrm{E}^{2}$, Pappas $\mathrm{Y}^{1}$ and Randhawa $\mathrm{G}^{1}$ \\ ${ }^{1}$ Institute for Health Research, University of Bedfordshire, UK \\ ${ }^{2}$ Senior Lecturer Health, Wellbeing and Social Care, Faculty of Wellbeing, The Open University, Milton Keynes, UK \\ *Corresponding author: Vseteckova J, Senior Lecturer Health, Wellbeing and Social Care, Faculty of Wellbeing, The Open \\ University, Milton Keynes, UK
}

\begin{tabular}{|c|c|}
\hline ARTICLE INFO & ABSTRACT \\
\hline 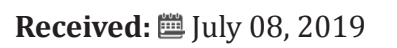 & Citation: Mahon A, Vseteckova J, Tilley E, Pappas Y, Randhawa G. A Systematic Scoping \\
\hline Published: 㓞 July 15, 2019 & $\begin{array}{l}\text { Review of the Physical and Mental Wellbeing Needs of Ageing Carers of Individuals with } \\
\text { Learning Disabilities within the United Kingdom. Biomed J Sci \& Tech Res 19(4)-2019. } \\
\text { BJSTR. MS.ID.003337. }\end{array}$ \\
\hline
\end{tabular}

\section{Introduction}

The population of the United Kingdom (UK) is getting older [1]. It was reported that in 2016, $18 \%$ of the UK population was aged 65 and over, with $2.4 \%$ aged 85 and over [1]. Furthermore, it is projected that by 2036 , over half of all local authorities in the UK will have $25 \%$ or more of their local population aged 65 and over. Concurrent with the fact that the general population of the United Kingdom's is projected to further increase, in addition to its ageing population the UK is also entering a period where a significant number of people with Learning Disabilities (LD) are living longer for the first time [2,3]. Crucially, most individuals with LD are cared for by family member, usually a parent. While the UK government have focused on increasing the provision of care for a general ageing population, little research has been conducted on the needs and/or support available for the increasing number of ageing careers. Particularly, little is known about the physical and mental wellbeing needs of individuals aged 65 years and older who care for a family member with learning disabilities. While it has been recognized these individuals require additional support, it is unclear exactly what support is needed and what interventions, if any, are currently available to support the physical and mental wellbeing needs of these ageing carers within the UK.

A preliminary search for existing scoping reviews and/or systematic reviews on the topic was conducted. Within the UK there are limited primary research papers and research syntheses available on this topic. To the authors knowledge no existing scoping reviews or systematic reviews on this topic currently exist. The aim of the current scoping review is to provide a greater understanding of the physical and mental wellbeing needs of ageing carers of people with learning disabilities within the UK as well as the interventions available to address these needs. This will include identifying the barriers and facilitators that can influence access to interventions as well as determining the outcomes, if any, that are reported as a consequence of interventions. Following the PICO framework [4], this scoping review aims to synthetize existing knowledge, identify gaps in the literature and provide recommendations for future research, which may lead to improved interventions and the potential to improve the healthcare of ageing carers.

The limited availability of UK-based primary research, and consequential gap in existing knowledge, resulted in the current scoping review. The aim of the current review is to collect and synthesize current knowledge on the physical and mental wellbeing 
needs of ageing carers (65 years old and over) for individuals with intellectual disability(ies) in the UK. Specifically, it aimed to:

i) Identify gaps in the literature,

ii) Provide recommendations for future research and

iii) Identify any interventions currently available to address career needs.

\section{Methods}

A systematic scoping review was deemed the most suitable method due to the research questions proposed by the current review and the literature it intended to include. The current review aimed to identify the physical and mental wellbeing needs of ageing carers for individuals with LD as well as mapping the interventions available to address those needs. Preliminary searches suggested that research on the topic of needs and interventions for ageing carers of individuals with learning disabilities within the United Kingdom is limited. While systematic reviews answer questions relating to specific interventions, the strict parameters required by this methodology deem it inappropriate for the purpose of the current review. Crucially, systematic scoping reviews are designed to synthesize knowledge according to an exploratory research question [5] and may include a wide variety of different research methodologies, allowing it to provide an overview of broad research fields such as that included in the current review [6]. Furthermore, in line with the aims of the current review, scoping reviews may include any gaps in current research, make recommendations for future research as well as mapping key concepts and bodies of literature [5] It is important to note that while scoping reviews generally do not require quality assessments of literature included, they do share a similar methodology to systematic reviews and as such they are rigours, transparent and methodical in their approach [7-9].

As an extension of the Preferred Reporting Items for Systematic Reviews and Meta-analysis (PRISMA) statement called the Preferred Reporting Items for Systematic Reviews and MetaAnalysis: extension for Scoping Reviews (PRISMA-ScR) is currently under development [10], the current scoping review followed the methodological framework proposed by Arksey and 0'Malley [7] as well as the amendments to this framework proposed by Levac, Colquhoun and O'Brien [11], the Joanna Briggs Institute [12] and the guidelines for best practices provided by Colquhoun et al. [13]. The Joanna Briggs Institute (JBI) Methodology for Scoping Reviews, will be "congruent with the PRISMA-ScR checklist" and "will assist in standardizing future scoping reviews". It has also been recommended that scoping review protocols follow the relevant aspects of the Preferred Reporting Items for Systematic Reviews and Meta-analysis for Protocols (PRISMA-P) guidelines [14] thus, the PRISMA-P was used to draft the current protocol [13].

The Arksey and O'Malley's scoping review framework consists of five stages: a. Identifying the research question,

b. Identifying relevant studies,

c. Study selection,

d. Charting the data,

e. Collating, summarizing and reporting results.

Each stage is discussed in further detail below. The last optional stage, consultation, was not included in the current scoping review.

\section{Stage 1: Identifying the Research Question}

The aim of the current scoping review is to provide a greater understanding of the physical and mental wellbeing needs of ageing carers of people with learning disabilities within the UK as well as the interventions available to address these needs. This included identifying the barriers and facilitators that can influence access to interventions as well as determining the outcomes, if any, for carers who access these interventions. Following the PICO framework, this scoping review aimed to synthetize existing knowledge, identify gaps in the literature and provide recommendations for future research, which may lead to improved interventions with the potential to improve the healthcare of ageing carers.

To meet these objectives, this review asked the following questions:

a. What are the physical and mental wellbeing needs of (unpaid) ageing carers for people with learning disabilities within the UK?

b. What interventions are available within the UK to address the identified physical and mental wellbeing needs of (unpaid) ageing carers for people with learning disabilities?

c. What outcomes, if any, are provided by interventions that are available within the UK to address the identified physical and mental wellbeing needs of (unpaid) ageing carers for people with learning disabilities?

d. What are the key barriers and facilitators affecting (unpaid) ageing carers for people with learning disabilities in accessing interventions within the UK?

\section{Stage 2: Identifying Relevant Studies}

Scoping reviews provide an overview of broad research fields [6] as such the current review used a wide and diverse search to source all relevant studies. This included a search of electronic databases, reference lists (ancestor searching), website organizations and conference proceedings. Articles and evaluation reports related to the topic of ageing carers of individuals with learning disabilities were identified through an initial exploratory online search using the electronic databases MEDLINE (PubMed) and CINAHL. The text words in the title and abstract of relevant retrieved papers were then analyzed as well as the index terms used to describe 
the articles. All identified keywords and index terms were used to develop a rigorous search strategy that was undertaken across all included databases. The reference list of identified reports and articles was also searched for additional studies. The reviewers' contacted authors of primary studies or reviews for further information, where relevant. The search was limited to literature written in English. No date restrictions were applied. The search strategy and details of the search dates can be found in Appendix 1.

\section{Inclusion Criteria}

\section{Types of Participants: Eligible participants included:}

i) Carers aged 65 and over for individuals (adults; aged 18+) with Learning Disabilities (LD).

ii) Carers must not be caring for the individual with learning disabilities as part of their professional vocation i.e. they will be unpaid parents, family members or friends.

iii) Providers and/or stakeholders involved in the provision of services specifically related to the physical and/or mental wellbeing of ageing carers of individuals with LD.

\section{Concept}

\section{Types of Outcome Measures}

To be included in the review, records had to report at least one of the following outcome measures:

\section{A. Primary Outcomes}

The primary outcomes of interest are the physical and mental wellbeing needs of ageing carers for individuals with learning disabilities, within the United Kingdom. It was expected that outcomes are diverse and context-specific, therefore, it was not possible to produce an exhaustive list at the outset. However, based on the review of the relevant literature, examples of primary outcomes regarding the physical and mental wellbeing needs of ageing carers may include:

1. Physiological needs (e.g. age-related disease onsets, age related physiological issues such as arthritis or joint replacements, raised blood pressure and raised blood glucose level),

2. Psychological needs (e.g. poor mental wellbeing including stress, depression, financial worries, fear of the future and anxiety. These needs can be quantitative self-reports or measured using psychological assessment tools such as the Warwick-Edinburgh mental well-being scale (WEMWBS) and the Hospital Anxiety \& Depression scale (HAD),

3. Social needs (e.g. social isolation, lack of new friendships and/or sense of belonging).

\section{B. Secondary Outcomes}

The secondary outcomes of interest are the availability of interventions to address the needs identified in the primary outcomes, including the facilitators and barriers affecting access, and the outcomes for service users, including personal, social, wellbeing, and health-related outcomes. Any interventions or any form of support available will be plotted in accordance with the primary care needs they address. Facilitators and barriers to accessing interventions may include: availability of interventions, awareness of available resources, concerns regarding the care needs of the individual they are caring for, provision of respite, finances and transport. The outcomes are expected to be diverse and context-specific and, therefore, can be measured in ways that include but are not limited to:

1. Physiological effects (e.g. any reported changes in agerelated disease, blood pressure and/or blood glucose level),

2. Psychological effects (e.g. any reported changes in carers mental well-being including stress, depression, financial worries, fear of the future and anxiety. These needs can be quantitative self-reported or measured using psychological assessment tools),

3. Social effects (e.g. any reported changes in carers social isolation, friendships and/or sense of belonging).

\section{Types of Interventions}

The aim of this systematic scoping review is to synthesize knowledge regarding all available information related to the exploratory research questions, as such all interventions will be included.

\section{Context/Setting}

Both carers and the individuals they care for must live within the United Kingdom. If the carer or the individuals, they are caring did not live in the United Kingdom they were excluded. Interventions that were implemented in the UK were included in the review. Those interventions that were implemented outside of the UK were excluded.

\section{E. Types of Studies}

The purpose of the current scoping review was to synthesize all relevant available knowledge. To provide a comprehensive overview of this research topic all existing literature was included, e.g. primary research studies, systematic reviews, meta-analyses, letters, guidelines, websites etc.

\section{F. Electronic Searches}

The following electronic databases were searched:

1. CINAHL

2. British Nursing Index

3. Web of Science

4. Cochrane library

5. Medline 

6. PsychInfo
7. SocIndex
8. University of York Centre for Reviews and Dissemination (DARE, NHS EED, HTA)

9. JBI Database of Systematic Reviews and Implementation Reports,
10. PubMed
11. EPPI
12. Epistemonikos,

To identify relevant evaluations in UK settings, the websites of the following organisations were searched:
a. The Kings Fund
b. The Health foundation
c. NESTA
d. NICE
e. Nuffield Trust
f. Department of Health

Additionally, grey literature was searched in OpenGrey, Google, and Google Scholar. The search terms "ageing carers of learning disabilities" and "older carers of learning disability" were used to identify grey literature because they were identified as the most relevant terms in the exploratory and database searches.

\section{G. Searching other Resources}

In addition, reference lists of all relevant studies, reviews, and reports were searched. Via contacting the British Library and other University libraries, the researcher obtained a full text PDF or an abstract containing sufficient details to determine eligibility of all potentially relevant studies.

\section{Stage 3: Study Selection}

Study Screening and Selection: Study selection (both at title/abstract screening and full text screening) was performed by three reviewers, independently. Any disagreements were solved by consensus or by the decision of a fourth reviewer were necessary. After eliminating the duplicates (studies that were identified more than once by the search engines), an initial screening of titles, abstracts, and summaries (if applicable) was undertaken to exclude records that clearly did not meet the inclusion criteria. Each record was classified as 'include' or 'exclude' to identify relevant and exclude irrelevant literature. The researchers were inclusive at this stage and, if uncertain about the relevance of a publication or report, it was left in. The full text was obtained for all the records that potentially met the inclusion criteria (based on the title and abstract/summary only). In a second step, all the full text papers were screened against the inclusion criteria, using a standardized tool. Studies that did not meet the inclusion criteria were listed with the reasons for exclusion. Multiple publications and reports on the same interventions were linked together and compared for completeness. The record containing the most complete data on any single intervention was identified as the primary article in the review, which was usually the original study or most recent evaluation report. An adapted PRISMA (Preferred Reporting Items for Systematic Reviews and Meta-analyses) flow-chart of study selection was included in the review [15].

\section{Stage 4: Charting the Data}

Data Extraction and Management: Data for analysis were extracted from the included studies and managed in an Excel spread sheet. A data extraction sheet was developed which was tailored to the requirements of the review. The data extraction sheet was tested on three included papers and, where necessary, it was revised to ensure it can be reliably interpreted and could capture all relevant data from different study designs. Extracted data included authors, year of study/report, aim/purpose, type of paper (e.g. journal article, annual evaluation report, etc), geographical area, study population (e.g., age of participants and learning disability diagnosed), sample size, study design, mental wellbeing needs identified (psychological and social), physical wellbeing needs identified, description of the interventions/services/support for mental wellbeing needs, description of the interventions/services/support for physical wellbeing needs, duration of interventions, factors that facilitate and/or hinder access to interventions/services/support, outcomes reported by carers accessing/not accessing interventions (and how measures), key findings that relate to the scoping review questions.

\section{Stage 5: Collating, Summarizing and Reporting the Results}

Presentation of the Results (Data Synthesis): Findings from included studies were synthesized narratively. The 'Guidance on the Conduct of Narrative Synthesis in Systematic Reviews' was used to advise the narrative synthesis [16]. First, a preliminary synthesis was conducted to develop an initial description of the findings of included records and to organize them so that patterns across records could be identified. In a second step, thematic analysis was used to analyze the findings. The following five steps of thematic analysis were followed adopting a recursive process [17]:

a) Familiarization with the extracted data

b) Generation of initial codes

c) Searching for themes

d) Reviewing themes

e) Defining and naming themes

Depending on the findings available the reviewers will aim to provide a flow chart mapping the interventions available for ageing carers of individuals with learning disabilities, for the needs that were identified. This review will highlight the needs of older caregivers for individuals with intellectual disability(ies) 
as well as the need for more high-quality research in this field. The information presented in this review may be considered, in the future, by primary care providers and funding bodies when planning future support for this growing population of carers.

\section{References}

1. Ranall M (2017) An overview of the UK population, how it's changed, what has caused it to change and how it is projected to change in the future. The UK population is also compared with other European countries.

2. Walker C (2015) Ageing and people with learning disabilities: in search of evidence. British Journal of Learning Disabilities 43(4): 246-253.

3. Walker C, Ward C (2013) Growing older together: Ageing and people with learning disabilities and their family carers. Tizard Learning Disability Review 18(3): 112-119.

4. Richardson WS, Wilson MC, Nishikawa J, Hayward RS (1995) The wellbuilt clinical question: A key to evidence-based decisions. ACP journal club 123(3): A12.

5. Colquhoun HL, Levac D, O’Brien KK, Straus S, Tricco AC, et al. (2014) Scoping reviews: time for clarity in definition, methods, and reporting. J Clin Epidemiol 67(12): 1291-1294.

6. Moher D, Shamseer L, Clarke M, Ghersi D, Liberati A, et al. (2015) Preferred Reporting Items for Systematic Review and Meta-Analysis Protocols (PRISMA-P) 2015 statement. Systematic reviews 4(1): 1.

7. Arksey H, O'Malley L (2005) Scoping studies: Towards a methodological framework. Int J Soc Res Methodol 8(1): 19-32.

8. Halas G, Schultz AS, Rothney J, Goertzen L, Wener P, et al. (2015) A scoping review protocol to map the research foci trends in tobacco control over the last decade. BMJ open 5(1): e006643.

\section{ISSN: 2574-1241}

DOI: 10.26717/BJSTR.2019.19.003337

Vseteckova J. Biomed J Sci \& Tech Res

This work is licensed under Creative Commons Attribution 4.0 License

Submission Link: https://biomedres.us/submit-manuscript.php
9. Pham MT, Rajić A, Greig JD, Sargeant JM, Papadopoulos A, et al. (2014) A scoping review of scoping reviews: advancing the approach and enhancing the consistency. Research synthesis methods 5(4): 371-385.

10. Tricco A, Strauss S, Moher D (2015) Preferred Reporting Items for Systematic Reviews and Meta-Analysis: extension for Scoping Reviews (PRISMA-ScR). Equator Network.

11. Levac D, Colquhoun H, O’Brien KK (2010) Scoping studies: Advancing the methodology. Implement Sci 5: 69.

12. Peters Micah, Godfrey Christina, McInerney Patricia, Soares Cassia, Khalil Hanan, et al. (2015) The Joanna Briggs Institute reviewers' manual 2015: Methodology for JBI scoping reviews. p. 24.

13. Colquhoun H (2016) Current best practices for the conduct of scoping reviews. EQUATOR Network.

14. Shamseer L, Moher D, Clarke M, Ghersi D, Liberati A, et al. (2015) Preferred Reporting Items for Systematic Review and Meta-Analysis Protocols (PRISMA-P) 2015: Elaboration and explanation. BMJ 349: g7647.

15. Moher D, Liberati A, Tetzlaff J, Altman DG, Prisma Group (2009) Preferred reporting items for systematic reviews and meta-analyses: The PRISMA statement. PLoS medicine 6(7): e1000097.

16. Popay J, Roberts H, Sowden A, Petticrew M, Arai L, et al. (2006) Guidance on the conduct of narrative synthesis in systematic reviews: A product from the ESRC methods programme. Version 1: b92.

17. Braun V, Clarke V (2006) Using thematic analysis in psychology. Qualitative research in psychology 3(2): 77-101.

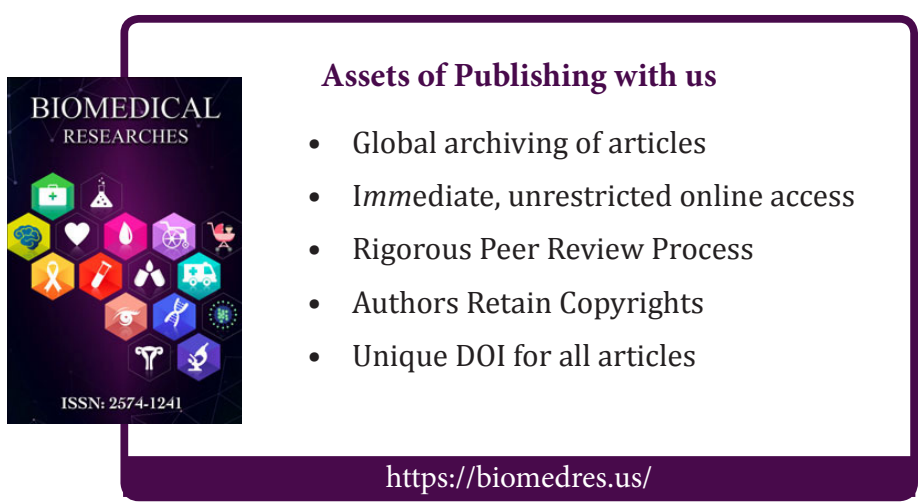

